\section{Correlation of lipid layer thickness measurements with fluorescein tear film break-up time and Schirmer's test}

\begin{abstract}
Purpose This study correlates measurement of lipid layer thickness (LLT) with two frequently used dry eye tests, fluorescein break-up time (FBUT) and Schirmer's test with anaesthesia (STA).

Methods Subjects ( $n=44$ eyes) with symptoms of dry eye and positive results for dry eye with either FBUT or STA or both were selected. Quantification of LLT was performed by the observation of colour interference patterns in zones of specular reflection using a custom-designed instrument.
\end{abstract}

Results All correlations among pairs of tests were strong and exhibited a significance of $P<0.000$ : STA with FBUT, Pearson's correlation 0.653; STA with LLT, 0.764; FBUT with LLT, 0.751. When LLT was high, ie $\geq 120 \mathrm{~nm}$, which occurred in 14 eyes, STA was also elevated in those eyes and FBUT was high in 13 of the 14 eyes. When LLT was low, ie $\leq 60$, which occurred in 22 eyes, STA was below normal in 14 of the 22 eyes, and FBUT was below normal in 15 of the 22 eyes. These clinical observations paralleled the statistical findings computed from the entire data set.

Conclusions The correlations demonstrated in this study support the premise (1) that measurement of LLT is a reliable test for the diagnosis of dry eye, and (2) that aqueous deficiency and lipid deficiency, as they apply to dry eye disorders, are not mutually exclusive.

Eye (2003) 17, 79-83. doi:10.1038/

sj.eye. 6700224

Keywords: lipid layer thickness; fluorescein tear film break-up time; Schirmer's test; dry eye
MA Isreb ${ }^{1}$, JV Greiner ${ }^{1,2}$, DR Korb ${ }^{3}$, T Glonek ${ }^{4}$, SS Mody ${ }^{1}$, VM Finnemore ${ }^{3}$ and CV. Reddy ${ }^{1}$

\section{Introduction}

The lipid layer is the most anterior layer of the preocular tear film ${ }^{1}$ and is vitally important to tear film function and stability. ${ }^{2}$ It retards water evaporation from the precorneal and corneal tear film surfaces. ${ }^{2}$ Lipid layer thickness (LLT), initially estimated to be $100 \mathrm{~nm}$ in the open eye, has been reported to vary from 13 to $170 \mathrm{~nm}^{4}$ The lipid layer is principally derived from the oils in the meibomian glands, ${ }^{5-10}$ with blinking spreading the lipids over the tear film. ${ }^{11}$ The LLT is dependent on several major factors including meibomian gland function, ${ }^{1}$ composition of meibomian gland secretions, ${ }^{5}$ effectiveness of blinking, ${ }^{12}$ width of the interpalpebral fissure, ${ }^{13}$ and ambient humidity. ${ }^{14}$ Numerous methods of utilizing interference patterns have been used to measure LLT, including slit lamp using a gooseneck lamp, ${ }^{15}$ a semiquantitative method, ${ }^{16}$ biodifferential interference microscopy, ${ }^{17,18}$ diffuse cold cathode tube illumination, ${ }^{4,19}$ differential interference contrast microscopy, ${ }^{20}$ Polaroid filters, ${ }^{19,21}$ and monochromatic light. ${ }^{22}$ Measurements of LLT by interference patterns have been correlated with tear film evaporation, ${ }^{23}$ tear film break-up time, ${ }^{23,24}$ and dry eye symptoms. ${ }^{24,25}$

The purpose of this study was to establish the correlation between LLT and two frequently used standard dry eye tests, ${ }^{26}$ fluorescein breakup time (FBUT) and Schirmer's test with anaesthesia (STA).

\section{Methods}

A symptoms questionnaire was used to select 35 subjects with complaints of dry eye.

Following a complete ophthalmic examination, subject inclusion depended on the following criteria:
${ }^{1}$ Schepens Eye Research Institute

Boston, MA, USA

${ }^{2}$ Department of Ophthalmology Harvard Medical School Boston, MA, USA

${ }^{3}$ Ocular Research of Boston Boston, MA, USA

${ }^{4}$ OMM Department Midwestern University Downers Grove, IL, USA

Correspondence: Dr Korb 100 Boylston St. Boston, MA 02116, USA Tel: 0016174236370 Fax: 0016174827373 E-mail: Drkorb@aol.com

Received: 26 June 2001 Accepted in revised form: 8 March 2002 
(1) absence of overt ocular disease, and

(2) a positive test result for dry eye with either FBUT or STA or both. After informed consent and a complete explanation of the proceedings, which conformed to the principles of the Declaration of Helsinki, subjects were examined in a room with dim light, ambient room temperature $\left(24-25^{\circ} \mathrm{C}\right)$, and a humidity range of $35-40 \%$.

\section{Symptoms questionnaire}

A questionnaire asked whether any dryness, grittiness or scratchiness, soreness, burning, watery eyes, or sensitivity to light were experienced. If any of these symptoms were present, the patient was asked to classify the frequency of the symptoms as sometimes, often, or constantly. Grading was as follows: one point for sometimes, two points for often, and three points for constantly. Subjects were included in the study if a score of three or more points was achieved.

\section{Measurement of lipid layer thickness (LLT)}

Examination of the lipid layer was achieved by studying optical interference patterns of the preocular tear film. LLT was measured using the method originally described by Guillon ${ }^{4,19}$ and then modified by Korb et al. ${ }^{12}$ Briefly, this method utilized a customized designed hemicylindrical broad-spectrum illumination source with heat-absorbing filters, a Zeiss binocular microscope with a beam splitter providing $70 \%$ light to a highresolution video camera, an SVHS recorder, and a high-resolution 20-in colour monitor. ${ }^{12}$ This instrument illuminated a $2.5 \mathrm{~mm}$ vertical and $5.0 \mathrm{~mm}$ horizontal area of the tear film, from the inferior limbus approaching the pupillary margin. This field provides an adequate area of observation without the light source directly entering the pupil, thus avoiding discomfort and potential reflex tearing. Analysis of the tear film lipid layer is based upon the observation of colour interference effects in zones of specular reflection at the air/lipid layer. The magnification of the colour interference patterns, as viewed in real time on the monitor, was varied from $10 \times$ to $25 \times$ for accurate analysis.

Trueness of colour representation on the video monitor was controlled by calibrating the instrument with Eastman Kodak colour reference standards (Wratten filters ${ }^{12}$ prior to analysis.

Quantification of LLT was performed by the observation of colour interference patterns in zones of specular reflection. ${ }^{15-21}$ For any given LLT, the colours of the interference patterns observed on the colour monitor are dependent upon the characteristics of the light
Table 1 Quantification and standardization of lipid layer thicknesses according to the dominant colour in the interference pattern

\begin{tabular}{lcc}
\hline Color & LLT (nm) & $\begin{array}{c}\text { Munsell color } \\
\text { standard notation }\end{array}$ \\
\hline White & 30 & $\mathrm{~N} \mathrm{9/0}$ \\
Grey (white) & 45 & $\mathrm{~N} 8.5 / 0$ \\
Grey (yellow) & 60 & $\mathrm{~N} 8 / 0$ \\
Grey (yelo & 10 YR 9/2 \\
Yellow & 75 & 10 YR 9/4 \\
Yellow (brown) & 90 & 7.5 YR $8 / 6$ \\
Brown (yellow) & 105 & $7.5 \mathrm{YR} 7 / 10$ \\
Brown & 120 & $2.5 \mathrm{YR} 6 / 10$ \\
Brown (blue) & 135 & $7.5 \mathrm{~PB} 8 / 2$ \\
Blue (brown) & 150 & $2.5 \mathrm{~PB} 7 / 4$ \\
Blue & 165 & $2.5 \mathrm{~PB} 6 / 4$ \\
\hline
\end{tabular}

source, the elements of the system used for imaging the interference patterns on the colour monitor, and the sensitivity of the eye of the observer. The judgment of the precise colour by the observer, and the coordination of multiple observers, requires colour standards. Colour attributes of hue, value, and chroma, as provided in the Munsell Book of Color, Glossy Finish Collection (Gretagmacbeth LLC, Munsell Color Services, New Windsor, NY 12553) were used to define the colour standards. Individual standards from the Munsell Book of Color were compared to the coloured interference pattern images on the monitor, and were then used as standards. The notation for the Munsell colour standard selected for each of the 11 colours is provided in Table 1. The thickness values assigned to specific observed colours were derived from previous work on the observation of interference colours from the tear film lipid layer (Table 1). ${ }^{12,15,16,19}$ The thickness assigned to the lipid layer corresponds to that represented by the dominant colour within the observation area. Interference patterns were recorded for each subject and then interpreted at different times by two independent examiners, and the average for each statistical case was computed.

\section{Measurement of fluorescein tear film break-up time (FBUT)}

The FBUT is an indirect measure of tear film stability, with instability indicated by a break-up time of $<10 \mathrm{s.}^{27}$ FBUT was measured following a $10 \mathrm{~min}$ rest interval using $2 \%$ sodium fluorescein solution that was diluted from 10\% Ak-FLOUR (Akron Inc., Buffalo Grove, IL). An Eppendorf ultra-microdigital pipette model \#4710 $(0.5-10 \mu \mathrm{l})$ and ultra-microtips $(0.5-10 \mu \mathrm{l})$ (Brinkman Instruments Inc., Westbury, NY) were used to instill $2 \mu \mathrm{l}$ 
of $2 \%$ fluorescein solution onto the inferior palpebral conjunctiva after gentle depression of the lower eyelid. ${ }^{28}$ When the fluorescein dye was expressed from the micropipette, a droplet formed which was held to the pipette tip by surface tension. This droplet was then brought near the palpebral conjunctiva of the depressed lower eyelid, and the dye was released from the micropipette tip when the liquid made contact with the tear film, being careful to avoid making contact with any ocular surface tissues. The lower eyelid was then slowly released. The subject was then asked to blink gently, but completely, three times. The tear film was then examined with a broad beam and the cobalt blue filter of a HaagStreit slit lamp. The interval between the last blink and the appearance of the first precorneal hypofluorescent spot, streak, or other irregularity interrupting the normal homogenous fluorescein pattern was recorded as the FBUT (seconds).

\section{Method of Schirmer's test with anaesthesia (STA)}

The Schirmer's test measures tear secretion, ie tear flow, with a result of $\leq 5 \mathrm{~mm}$ indicating impaired secretion. ${ }^{29,30}$ While the Schirmer test has been reported as 'notoriously variable and fraught with sources of error' ${ }^{31}$ it remains the universal test for the evaluation of the production of tears by the lacrimal glands. Since the primary problem with the Schirmer test is the variable and unknown level of reflex tearing, we elected to follow the recommendation to use topical anaesthetic to minimize excess reflex tearing. ${ }^{31,32}$ We have found that the use of topical anaesthesia and then blotting the excess tears from the inferior fornix provides the best opportunity to attain repeatability of Schirmer's test results.

Sixty (60) minutes after FBUT testing, STA was performed by instilling one drop of proparacaine $0.5 \%$ anaesthetic, waiting for $4 \mathrm{~min}$, then instilling a second drop. After a period of $1 \mathrm{~min}$, the lower lid was gently depressed exposing the lower fornix. Sterile gauze pads (Johnson \& Johnson) were fashioned in the shape of a wick using sterile gloves. The wick was positioned between the lower fornix and lower eyelid margin and used to blot excess tears while the lower lid was gently released and allowed to return to the anatomic position. After $3 \mathrm{~s}$, the lower lid was again gently depressed and the absorbent wick carefully removed. This blotting procedure of the inferior lacrimal lake was repeated twice. The lower eyelid was again gently depressed and Schirmer's test paper (Whatman \#15 filter paper) was placed at the junction between the middle and outer third of the lower eyelid, with care not to irritate the cornea or the conjunctiva. The subject was then instructed to close the eyelids gently, not to speak or make facial expressions that could result in the movement of the facial musculature, and to position the chin on the chin rest of the slit lamp. This was done to maintain the subject's head in an erect position. The subject was also instructed not to move the eyes in order to minimize or avoid contact between the cornea and Schirmer's test paper. All of the above precautions were taken to minimize reflex tearing. STA results were then recorded after $5 \mathrm{~min}$. The wet portion of the paper was measured in millimetres $(\mathrm{mm} / 5 \mathrm{~min})$.

\section{Data analysis}

Results of LLT, FBUT and STA of all subjects ( $n=44$ eyes) were correlated to each other using the Pearson's correlation $\left(n=44\right.$ eyes). ${ }^{33}$ A probability of $P<0.05$ was accepted as significant.

\section{Results}

Of the 35 subjects, 8 male and 14 female subjects (age 2275 years; mean $43 \pm 16 \mathrm{SD}$ ) met the inclusion criteria. The remaining 13 subjects, although scoring 3 or more points on the symptoms questionnaire, demonstrated thick lipid layers and normal FBUT and STA values and were therefore not included in the study.

The results demonstrate a good correlation between LLT and each of the other two tests, FBUT and STA (Table 2). The lipid layer thickness varied from 45 to $135 \mathrm{~mm}$ with an average of $89.77 \pm 42.51 \mathrm{SD}$, break-up time from 3 to $16 \mathrm{~s}$ with an average of $8.59 \pm 4.24 \mathrm{SD}$, and Schirmer's test with anaesthesia from 2 to $21 \mathrm{~mm} / 5 \mathrm{~min}$ with an average of $9.43 \pm 5.68 \mathrm{SD}$. The positive correlations among LLT, FBUT, and STA were firm and exhibited high probability (Table 2).

Using the clinical classification in Table 3, there are high levels of correlation at both ends of the LLT spectrum. When LLT was high, ie $\geq 120$ which occurred in 14 eyes, STA was also elevated in these eyes and FBUT was elevated in 13 of the 14 eyes. When LLT was low, ie $\leq 60$, which occurred in 22 eyes, STA was below normal in 14 of the 22 eyes, and FBUT was below normal in 14 of the 22 eyes. These clinical observations based on

Table 2 Correlations among lipid layer thickness (LLT), fluorescein break-up time (FBUT), and Schirmer's test with anaesthesia (STA)

\begin{tabular}{llcc}
\hline Correlation parameters & $\begin{array}{c}\text { Correlation } \\
\text { coefficient } \\
\text { (Pearson's) }\end{array}$ & Probability \\
\hline STA & FBUT & 0.6533 & 0.000 \\
STA & LLT & 0.7639 & 0.000 \\
FBUT & LLT & 0.7506 & 0.000 \\
\hline
\end{tabular}


Table 3 A classification of probability of dry eye based on fluorescein break-up time (FBUT), Schirmer's test with anesthesia (STA), and lipid layer thickness (LLT) measurements

\begin{tabular}{lccc}
\hline Category & FBUT $(\mathrm{s})$ & STA $(\mathrm{min})$ & LLT $(\mathrm{nm})$ \\
\hline I & $0-5$ & $0-5$ & $\leq 60$ \\
II & $6-<10$ & $6-<10$ & $\geq 75-105$ \\
III & $\geq 10$ & $\geq 10$ & $\geq 120$ \\
\hline
\end{tabular}

Category I=a high probability of having (significant) dry eye signs and symptoms.

Category II=an intermediate probability of having dry eye signs and symptoms.

Category III=a low or zero probability of having dry eye signs and symptoms.

individual cases (eyes) (Table 3) parallel the statistical findings computed from the entire data set (Table 2).

\section{Discussion}

Thicker lipid layers $(\geq 120 \mathrm{~nm})$ are desirable and are correlated to optimal tear film stability and tear film characteristics. $^{23,24}$ Thin lipid layers $(\leq 60 \mathrm{~nm})$ have been correlated with tear film instability, ${ }_{1}^{23,24}$ increased evaporation rate, ${ }^{23}$ high osmolality, ${ }^{34}$ symptoms, ${ }^{24,25}$ and dry eye disorders. ${ }^{25}$ The correlation coefficients in this study for LLT with STA and LLT with FBUT were higher than for STA with FBUT. These data support the premise that measurement of LLT is as valid as either FBUT or STA for the diagnosis of dry eye disorders in those instances of patients presenting with symptoms suggestive of dry eye conditions.

The data suggest that a thin LLT results in an aqueous tear-deficient state and an unstable tear film; however, one could also conclude that an aqueous tear-deficient state would result in a thin lipid layer. Conventional literature on dry eye distinguishes between aqueous- and lipid-deficient tear states. ${ }^{30}$ Aqueous-deficient tear states are usually considered a result of lacrimal gland disorder, while lipid-deficient states are usually considered a result from obstructive meibomian gland dysfunction with the sequelae of increased tear evaporation. ${ }^{35}$ The National Eye Institute (NEI) Workshop's definition of dry eye is '.... a disorder of the tear film due to tear deficiency or excessive tear evaporation which causes damage to the interpalpebral ocular surface and is associated with symptoms of ocular discomfort'. ${ }^{30}$ Considering the results presented herein, it would appear that the states of aqueous deficiency and lipid deficiency as they apply to dry eye disorders are not mutually exclusive.

In addition to the 22 subjects who met the criteria for admission to the study, the 13 additional subjects screened scored 3 or more points on the symptoms questionnaire, but had normal FBUT and STA findings, as well as a thick lipid layer. These subjects were not admitted into the study. These results suggest that the reported symptoms assumed to be indicative of dry eye were, however, not diagnostic of dry eye. Symptoms usually considered to be of dry eye aetiology may be due to other causes including allergies and environmental factors. Further, many vocational and other conditions, including computer use, concentrated near work and fatigue, may result in inhibition of blinking and the presence of dry eye symptoms. ${ }^{36-43}$ Thus, many dry eye states are likely to be intermittent and variable in severity, and may not be present or recalled at the time of the subject's examination, or when participating in a study. A more comprehensive and specific history and questionnaire is required to address this issue with emphasis on the presence of symptoms at the time of the examination. The correlations demonstrated in this study support the premise (1) that measurement of lipid layer thickness is a reliable test for the diagnosis of dry eye, and (2) that aqueous deficiency and lipid deficiency, as they apply to dry eye disorders, are not mutually exclusive.

\section{Acknowledgements}

We wish to thank Mary McCann and John McCann for their assistance with the calibration of the lipid layer thickness and colour standards.

\section{References}

1 Wolff E. The muco-cutaneous junction of the lid margin and distribution of the tear fluid. Trans Ophthalmol Soc UK 1946; 66: 291-308.

2 Mishima S, Maurice DM. The oily layer of the tear film and evaporation from the corneal surface. Exp Eye Res 1961; 1: 39-45.

3 McDonald JE. Surface phenomena of tear films. Trans Am Ophthalmol Soc 1968; 66: 905-939.

4 Guillon J-P. Tear film structure and contact lenses In: Holly FJ (ed). The Preocular Tear Film. Dry Eye Institute: Lubbock, TX, 1986, pp 914-939.

5 Tiffany JM. Composition and biophysical properties of the tear film: knowledge and uncertainty. Adv Exp Med Biol 1994; 350: 231-238.

6 Holly FJ, Lemp MA. Tear film physiology and dry eyes. Surv Ophthalmol 1977; 22: 69-87.

7 Brown SI, Dervichian DG. The oils of the meibomian glands. Arch Ophthalmol 1969; 82: 537-540.

8 Tiffany JM. The meibomian lipids of the rabbit. 1. Overall composition. Exp Eye Res 1979; 29: 195-202.

9 Tiffany JM, Marsden RG. The influence of composition on physical properties of meibomian gland secretion In: Holly FJ (ed). The Preocular Tear Film in Health, Disease, and Contact Lens Wear. Dry Eye Institute: Lubbock, TX, 1986, pp 597-608.

10 Nicolaides N. Recent findings on the chemical composition of steer and human meibomian glands In: Holly FJ (ed). 
The Preocular Tear Film in Health, Disease, and Contact Lens Wear. Dry Eye Institute: Lubbock, TX, 1986, pp. 570-576.

11 Brown SI, Dervichian DG. Hydrodynamics of blinking: in vitro study of the interaction of the superficial oily layer and the tears. Arch Ophthalmol 1969; 82: 541-547.

12 Korb DR, Baron DF, Herman IP et al. Tear film lipid layer thickness as a function of blinking. Cornea 1994; 13: 354-359.

13 Dilly PN. Structure and function of the tear film. Adv Exp Med Biol 1994; 350: 239-247.

14 Korb DR, Greiner JV, Glonek T et al. Effect of periocular humidity on the tear film lipid layer. Cornea 1996; 15: 129-134.

15 McDonald JE. Surface phenomena of the tear film. Arn J Ophthalmol 1969; 67: 5664.

16 Norn MS. Semiquantitative interference study of fatty layer of precorneal film. Acta Ophthalmol 1979; 57: 766-774.

17 Hamano H, Hori M, Kawabe M et al. Clinical applications of biodifferential interference microscope. Contact Intraocular Lens Med J 1980; 6: 229-235.

18 Hamano H, Hori M, Kawabe M et al. Biodifferential interference microscopic observations on anterior segment of eye. J Jpn Contact Lens Soc 1979; 21: 229-231.

19 Guillon J-P. Tear film photography and contact lens wear. J Br Contact Lens Assoc 1982; 5: 84-87.

20 Kilp H, Schmidt E, Kirchner L, Zipf-Pohl A. Tear film observation by reflecting microscopy and differential interference contrast microscopy In: Holly FJ (ed). The Preocular Tear Film in Health, Disease, and Contact Lens Wear. Dry Eye Institute: Lubbock, TX, 1986, pp 564-569.

21 Josephson JE. Appearance of the preocular tear film lipid layer. Am J Optom Physiol Opt 1983; 60: 883-887.

22 Doane MG, Lee ME. Tear film interferometry as a diagnostic tool for evaluating normal and dry eye tear film. Adv Exp Med Biol 1998; 438: 297-303.

23 Craig JP, Tomlinson A. Importance of the lipid layer in human tear film stability and evaporation. Optom Vis Sci 1997; 74: 8-13.

24 Guillon M, Styles E, Guillon JP, Maissa C. Preocular tear film characteristics of nonwearers and soft contact lens wearers. Optom Vis Sci 1997; 74: 273-279.

25 Korb DR, Greiner JV. Increase in tear film lipid layer thickness following treatment of meibomian gland dysfunction. Adv Exp Med Biol 1994; 350: 293-298.

26 Korb DR. Survey of preferred test for the diagnosis of the tear film and dry eye. Cornea 2000; 19: 483-486.
27 Lemp MA, Hamill JR. Factors affecting tear film break-up in normal eyes. Arch Ophthalmol 1973; 89: 103-105.

28 Finnemore VM, Korb DR, Greiner JV, Glonek T, Herman JP. Fluorescein dye concentration as a factor in tear film fluorescence. Adv Experimental Med Biol 1998; 438: 875-878.

29 V Bijsterveld OP. Diagnostic tests in the sicca syndrome. Arch Ophthalmol 1969; 82: 10-14.

30 Lemp MA. Report of the National Eye Institute/industry workshop on clinical trials in dry eyes. CLAO J 1995; 21: 221-232.

31 Abelson MB, Lamberts DW. Dry eye update. Excerpta Med (Amsterdam) 1983; 1-17.

32 Jones LT. The lacrimal secretory system and its treatment. Am J Ophthalmol 1966; 62: 47-60.

33 Norusis MJ. (ed). The SPSS Guide to Data Analysis for SPSS/ $P C+$, 2nd ed. SPSS: Chicago, 1991, pp 148-416.

34 Craig JP. The role of tear physiology in ocular surface temperature. Eye 2000; 14: 635-641.

35 Bron AJ. The Doyne Lecture. Reflections on the tears. Eye 1997; 11: 583-602.

36 Dainoff MJ, Happ A, Crane P. Visual fatigue and occupational stress in VDT operators. Hum Factors 1981; 23: 421-438.

37 Rossignol MA, Pechter ME, Summers VM, Pagnotto L. Video display terminal use and reported health symptoms among Massachusetts clerical workers. J Occup Med 1987; 29: $112-118$.

38 Tsubota K, Nakomori K. Dry eyes and video display terminals. N Engl J Med 1993; 328: 584.

39 Nakaishi H, Yamada Y. Abnormal tear dynamics and symptoms of eyestrain in operators of visual display terminals. Occup Environ Med 1999; 56: 6-9.

40 Yaginuma Y, Yamada H, Nagai H. Study of the relationship between lacrimation and blink in VDT work. Ergonomics 1990; 33: 799-809.

41 Patel S, Henderson R, Bradley L, Galloway B, Hunter L. Effect of visual display unit use on blink rate and tear stability. Optom Vis Sci 1991; 68: 888-892.

42 Ousler GW, Gomes PJ, Crampton HJ, Abelson MB. The effects of a lubricant eye drop on the signs and symptoms of computer vision syndrome (CVS) exacerbated in a controlled adverse environment. Invest Ophthalmol Vis Sci 1999; 40 (ARVO Suppl): B722.

43 Nakamori K, Odawara M, Nakajima T, Mizutani T, Tsubota $\mathrm{K}$. Blinking is controlled primarily by ocular surface conditions. Am J Opthalmol 1997; 124: 24-30. 